\title{
Linearized-moment analysis of the temperature jump and temperature defect in the Knudsen layer of a rarefied gas
}

\author{
Xiao-Jun $\mathrm{Gu}^{*}$ and David. R. Emerson \\ Scientific Computing Department, STFC Daresbury Laboratory, Warrington WA4 4AD, United Kingdom
}

(Received 26 March 2014; published 27 June 2014)

\begin{abstract}
Understanding the thermal behavior of a rarefied gas remains a fundamental problem. In the present study, we investigate the predictive capabilities of the regularized 13 and 26 moment equations. In this paper, we consider low-speed problems with small gradients, and to simplify the analysis, a linearized set of moment equations is derived to explore a classic temperature problem. Analytical solutions obtained for the linearized 26 moment equations are compared with available kinetic models and can reliably capture all qualitative trends for the temperature-jump coefficient and the associated temperature defect in the thermal Knudsen layer. In contrast, the linearized 13 moment equations lack the necessary physics to capture these effects and consistently underpredict kinetic theory. The deviation from kinetic theory for the 13 moment equations increases significantly for specular reflection of gas molecules, whereas the 26 moment equations compare well with results from kinetic theory. To improve engineering analyses, expressions for the effective thermal conductivity and Prandtl number in the Knudsen layer are derived with the linearized 26 moment equations.
\end{abstract}

DOI: 10.1103/PhysRevE.89.063020

PACS number(s): 47.10.ab, 51.10.+y, 47.45.Gx

\section{INTRODUCTION}

At the microscale, gaseous heat transfer no longer follows Fourier's law. Gases in microdevices frequently experience rarefaction effects as the molecular mean free path $\lambda$ is often comparable to the characteristic dimension of the device. Consequently, gas molecules undergo collisions with solid walls more often than among themselves in order to reach equilibrium. The state of a dilute gas can be described by either kinetic theory, which treats the gas as a large number of interacting molecules undergoing binary collisions according to prescribed laws, or hydrothermal dynamics, which employs macroscopic quantities and their governing equations to describe the gas behavior. The Boltzmann equation [1] and direct simulation Monte Carlo (DSMC) [2] are the two main kinetic methods used to simulate nonequilibrium gas flows, particularly when the flow velocity is large and nonequilibrium effects are strong. For flows at low speed and not far away from the equilibrium state, as those encountered in microelectromechanical systems (MEMS), both methods are computationally expensive for practical applications. Conversely, the traditional hydrothermodynamic Navier-Stokes-Fourier (NSF) equations, which govern the first five moments of the molecular distribution function, i.e., velocity, temperature, and pressure, are only accurate for gaseous flow close to the equilibrium state. When the gas departs from equilibrium, more macroscopic variables, or moments, are required to approximate the gas conditions accurately enough for engineering design.

The method of moments originally proposed by Grad [3] is an approximate solution procedure to the Boltzmann equation. In Grad's approach, the molecular distribution function $f$ is expanded in terms of Hermite polynomials, the coefficients of which are linear combinations of the moments of the distribution function. An infinite set of Hermite coefficients is equivalent to the distribution function itself and there is no loss

*Corresponding author: xiaojun.gu@stfc.ac.uk of kinetic information. In practice, however, the distribution function has to be truncated, and the specific problem to be addressed will determine the order of the truncation. The truncated distribution will be denoted as Grad's distribution function, $f_{G}$. The governing equations of the moments retained in $f_{G}$ can be derived from the Boltzmann equation [4]. In this approach, the Boltzmann equation is satisfied in a certain average sense rather than at the molecular distribution function level. However, it provides a useful bridge between kinetic theory and hydrothermal dynamics and provides a way to extend classical fluid dynamics to nonequilibrium flow.

In addition to the conservation laws, the moment method employs the governing equations for the deviatoric stress tensor, the heat flux, and higher moments of the molecular distribution function $f$ to describe the nonequilibrium state of a gas. With a finite number of moments, such as 13 or 26 moments in three-dimensional applications (the number of moments in one- or two-dimensional problems is much reduced), recent studies have shown that both the regularized 13 (R13) and 26 (R26) moment equations are able to capture several well known nonequilibrium phenomena, such as the bimodal temperature profile in force-driven Poiseuille flow, nongradient heat flux in Couette flow, and the Knudsen minimum [5-9]. They have also been used to study oscillatory flow with some success [10].

For many low-speed flows, it should be sufficient to use a linearized set of moment equations. In our previous study [9], the linearized R13 (LR13) and R26 (LR26) were used to study Kramers' problem. It was found that the LR26 equations can capture the hydrodynamic Knudsen layer fairly accurately in terms of the defect velocity profile and slip coefficient, while the LR13 equations do not provide sufficient accuracy, which is consistent with the study by Young [11]. In the present work, both the LR13 and LR26 equations are used to study thermal behavior in the early transition regime. The temperature-jump problem, as proposed by Smoluchowski [12], is the most basic configuration that allows the effect of a solid boundary to be investigated without the 
additional complications found in more realistic geometries. This problem has been extensively studied in kinetic theory by solving the linearized Boltzmann equation [13-16] and the data provides a useful numerical benchmark for the development of macroscopic models. However, no DSMC data is available for this classic temperature-jump problem. Analytical solutions of the temperature-jump problem are derived from the LR13 and LR26 moment equations and the temperature defect and temperature-jump coefficient are compared with numerical solutions from kinetic theory to assess the validity and accuracy of the moment equations. In this paper, we show that rarefaction effects can be described by the macroscopic governing equations to good accuracy and the physics of nonequilibrium gas flow is well embedded in the moment equations in the early transition regime.

\section{LINEARIZED-MOMENT EQUATIONS AND THE TEMPRATURE-JUMP PROBLEM}

Once the distribution function $f$ is known, the moments with respect to the particle's velocity $\boldsymbol{\xi}$ can be determined. For example, the density $\rho$ and the momentum $\rho u_{i}$ can be obtained from

$$
\rho=\int f d \xi
$$

and

$$
\rho u_{i}=\int \xi_{i} f d \xi,
$$

where $\xi_{i}$ and $u_{i}$ represent the particle and fluid velocity, respectively. It is convenient to introduce the intrinsic or peculiar velocity as $c_{i}=\xi_{i}-u_{i}$, so that the moments with respect to $c_{i}$ can be determined. A set of $N$ moments is then used to describe the state of the gas through

$$
\rho_{i_{1} i_{2} \ldots i_{N}}=\int c_{i_{1}} c_{i_{2}} \ldots c_{i_{N}} f d \xi
$$

Any moment can be expressed by its trace and traceless parts. For example, the pressure tensor can be separated as

$$
p_{i j}=\int c_{i} c_{j} f d \xi=p \delta_{i j}+p_{\langle i j\rangle}=p \delta_{i j}+\sigma_{i j},
$$

where $\delta_{i j}$ is the Kronecker delta function, $p=p_{k k} / 3$ is the pressure, and $\sigma_{i j}=p_{\langle i j\rangle}$ is the deviatoric stress tensor. The angular brackets are used to denote the traceless part of a symmetric tensor. Furthermore, the temperature $T$ is given by

$$
\frac{3}{2} \rho R T=\frac{1}{2} \int c_{k} c_{k} f d \xi .
$$

The heat flux vector is defined as

$$
q_{i}=\frac{1}{2} \int c_{k} c_{k} c_{i} f d \xi
$$

For the convenience of modeling, the higher moments are decomposed into values approximated with Grad's distribution function $\left.\rho_{i_{1} i_{2} \ldots i_{N}}\right|_{f_{G}}$, and the deviation from their true value. With Grad's 26 moment distribution function $f_{\mathrm{G} 26}$ the following moments used in the present study can be expressed by

$$
\begin{aligned}
\rho_{\langle i j k\rangle} & =m_{i j k}+\rho_{\langle i j k\rangle \mid f_{G 26}}=m_{i j k}, \quad \rho_{\langle i j k l\rangle}=\phi_{i j k l}+\rho_{\langle i j k l\rangle \mid f_{G 26}}=\phi_{i j k l}, \\
\rho_{\langle i j\rangle r r} & =R_{i j}+\rho_{\langle i j\rangle r r \mid f_{G 26}}=R_{i j}+7 R T \sigma_{i j}, \quad \rho_{r r\langle i j k\rangle}=\psi_{i j k}+\rho_{r r}\langle i j k\rangle \mid f_{G 26}=\psi_{i j k}+9 R T m_{i j k}, \\
\rho_{r r s s} & =\Delta+\rho_{r r s s \mid f_{G 26}}=\Delta+15 p R T, \quad \rho_{r r s s i}=\Omega_{i}+\rho_{r r s s i \mid f_{G 26}}=\Omega_{i}+28 R T q_{i},
\end{aligned}
$$

where $m_{i j k}, R_{i j}, \Delta, \psi_{i j k}, \phi_{i j k l}$, and $\Omega_{i}$ represent the difference between the true value of the higher moments and their corresponding approximation with $f_{G 26}$.

The derivation of the R13 and R26 moment equations for a monatomic gas of Maxwell molecules and their linearization can be found in Refs. [4,6,7,17,18], respectively. For the temperature-jump problem, the coordinates are chosen such that the wall is parallel to the $x$ direction and $y$ is the direction perpendicular to the wall which is located at $y=0$. In the absence of a temperature gradient, the pressure, temperature, and density of the equilibrium gas are $p_{0}, T_{0}$, and $\rho_{0}$, respectively. A temperature gradient normal to the wall is imposed as in kinetic studies [13-16]. All derivatives in the $x$ direction are zero and mass conservation is satisfied automatically. The energy conversation law is reduced to

$$
\frac{\partial \bar{q}_{y}}{\partial \bar{y}}=0
$$

Equation (8) indicates that the normal heat flux $\bar{q}_{y}$ is a constant in the whole domain. The linearized one-dimensional moment equations are decoupled into a velocity-related and temperature-related set. The equations involved in the classic temperature-jump problem, obtained from the LR26 moments with the aid of Eq. (8), are reduced to a five moment system for Maxwell molecules:

$$
\begin{aligned}
\frac{\partial \bar{m}_{y y y}}{\partial \bar{y}} & =-\sqrt{\frac{\pi}{2}} \frac{L}{\lambda} \bar{\sigma}_{y y}, \\
\frac{1}{2} \frac{\partial \bar{R}_{y y}}{\partial \bar{y}} & =-\frac{2}{3} \sqrt{\frac{\pi}{2}} \frac{L}{\lambda} \bar{q}_{y}-\frac{5}{2} \frac{\partial \bar{T}}{\partial \bar{y}}-\frac{\partial \bar{\sigma}_{y y}}{\partial \bar{y}}-\frac{1}{6} \frac{\partial \bar{\Delta}}{\partial \bar{y}}, \\
\frac{\partial \bar{\phi}_{y y y y}}{\partial \bar{y}} & =-\frac{3}{2} \sqrt{\frac{\pi}{2}} \frac{L}{\lambda} \bar{m}_{y y y}-\frac{9}{5} \frac{\partial \sigma_{y y}}{\partial \bar{y}}-\frac{9}{35} \frac{\partial \bar{R}_{y y}}{\partial \bar{y}}, \\
\frac{\partial \bar{\psi}_{y y y}}{\partial \bar{y}} & =-\frac{7}{6} \sqrt{\frac{\pi}{2}} \frac{L}{\lambda} \bar{R}_{y y}-2 \frac{\partial \bar{m}_{y y y}}{\partial \bar{y}}-\frac{4}{15} \frac{\partial \bar{\Omega}_{y}}{\partial \bar{y}}, \\
\frac{\partial \bar{\Omega}}{\partial \bar{y}} & =-\frac{2}{3} \sqrt{\frac{\pi}{2}} \frac{L}{\lambda} \bar{\Delta} .
\end{aligned}
$$


They are closed by the following constitutive relationships:

$$
\begin{aligned}
\bar{\phi}_{y y y y} & =-\frac{16}{7 Z} \frac{\lambda}{L} \sqrt{\frac{2}{\pi}} \frac{\partial \bar{m}_{y y y}}{\partial \bar{y}}, \\
\bar{\psi}_{y y y} & =-\frac{81}{35 Y} \frac{\lambda}{L} \sqrt{\frac{2}{\pi}} \frac{\partial \bar{R}_{y y}}{\partial \bar{y}},
\end{aligned}
$$

and

$$
\bar{\Omega}_{y}=-\frac{\lambda}{L} \sqrt{\frac{2}{\pi}}\left(\frac{7}{3} \frac{\partial \bar{\Delta}}{\partial \bar{y}}+4 \frac{\partial \bar{R}_{y y}}{\partial \bar{y}}\right),
$$

in which $Y=1.698$ and $Z=2.097$ are collision constants for Maxwell molecules. The variables with an overbar symbol are small dimensionless deviations from an equilibrium state given by $\rho_{0}, T_{0}$ (or $p_{0}$ ), and $u_{0}$, as defined in Ref. [18]:

$$
\begin{aligned}
T & =(1+\bar{T}) T_{0}, \quad m_{y y y}=\rho_{0}\left(R T_{0}\right)^{3 / 2} \bar{m}_{y y y}, \quad \psi_{y y y}=\rho_{0}\left(R T_{0}\right)^{5 / 2} \bar{\psi}_{y y y}, \\
\sigma_{y y} & =\rho_{0} R T_{0} \bar{\sigma}_{y y}, \quad R_{y y}=\rho_{0}\left(R T_{0}\right)^{2} \bar{R}_{y y}, \quad \Omega_{y}=\rho_{0}\left(R T_{0}\right)^{5 / 2} \bar{\Omega}_{y}, \\
q_{y} & =\rho_{0}\left(R T_{0}\right)^{3 / 2} \bar{q}_{y}, \quad \phi_{y y y y}=\rho_{0}\left(R T_{0}\right)^{2} \bar{\phi}_{y y y y}, \quad \Delta=\rho_{0}\left(R T_{0}\right)^{2}, \quad \bar{\Delta} y=L \bar{y} .
\end{aligned}
$$

The mean free path $\lambda$ is defined by

$$
\lambda=\frac{\mu}{p_{0}} \sqrt{\frac{\pi R T_{0}}{2}},
$$

where $\mu$ is the viscosity. For the temperature-jump problem and consistency with kinetic theory [13-16], the thermal characteristic length $L$ is given by

$$
L=\frac{2 \kappa}{5 p_{0}} \sqrt{\frac{2 T_{0}}{R}}
$$

in which $\kappa$ is the thermal conductivity. In the hydrodynamic limit, for a monoatomic gas with the Prandtl number, $\operatorname{Pr}$, the thermal conductivity is given by

$$
\kappa=\frac{5}{2} \frac{R \mu}{\operatorname{Pr}} .
$$

The ratio of the hydrodynamic mean free path, $\lambda$, to the characteristic thermal length, $L$, is

$$
\frac{\lambda}{L}=\frac{\sqrt{\pi}}{2} \operatorname{Pr} .
$$

For Maxwell molecules, the Prandtl number, $\operatorname{Pr}=2 / 3$, so that $\lambda / L=\sqrt{\pi} / 3$.

The wall boundary conditions are constructed from Maxwell's kinetic boundary treatment [7] and the relevant terms related to the temperature-jump problem are linearized as

$$
\begin{aligned}
\bar{T}= & -G \frac{\bar{q}_{y}}{2}-\frac{\bar{\sigma}_{y y}}{4}-\frac{75 \bar{R}_{y y}+28 \bar{\Delta}}{840} \\
& +\frac{\bar{\phi}_{y y y y}}{24}-\left(1-\frac{T_{w}}{T_{0}}\right) p_{b}, \\
\bar{\sigma}_{y y}= & -G\left(\frac{5 \bar{m}_{y y y}+6 \bar{q}_{y}}{10}\right)-\frac{\bar{R}_{y y}}{7}-\frac{\bar{\Delta}}{30}-\frac{\bar{\phi}_{y y y y}}{6} \\
& -\bar{T}-\left(1-\frac{T_{w}}{T_{0}}\right) p_{b}, \\
\bar{R}_{y y}= & -G\left(\frac{21 \bar{q}_{y}}{8}+\frac{35 \bar{m}_{y y y}}{16}+\frac{35 \bar{\psi}_{y y y}}{144}+\frac{3 \bar{\Omega}_{y}}{32}\right) \\
& -\frac{7}{2}\left(\bar{\sigma}_{y y}+\frac{\bar{\Delta}}{15}+\frac{\bar{\phi}_{y y y y}}{3}+\bar{T}\right)
\end{aligned}
$$

$$
\begin{aligned}
& +\frac{7\left(p_{b}-\bar{T}\right)}{4}\left(\frac{T_{w}^{2}}{T_{0}^{2}}-1\right) \\
\bar{\Delta}= & -\frac{35}{4} G\left(\bar{q}_{y}+\frac{\bar{\Omega}_{y}}{28}\right)-\frac{15}{4} \bar{\sigma}_{y y}-\frac{15}{8} \bar{R}_{y y}+\frac{35}{48} \bar{\phi}_{y y y y} \\
& -15 \bar{T}+\frac{15}{2}\left(p_{b}-\bar{T}\right)\left(\frac{T_{w}^{2}}{T_{0}^{2}}-1\right)
\end{aligned}
$$

in which

$$
p_{b}=1+\bar{p}+\frac{\bar{\sigma}_{y y}}{2}-\frac{30 \bar{R}_{y y}+7 \bar{\Delta}}{840}-\frac{\bar{\phi}_{y y y y}}{24},
$$

where $G=\alpha^{*} \sqrt{\pi / 2}, \alpha^{*}=(2-\alpha) / \alpha$, and $\alpha$ is the momentum accommodation coefficient, indicating that a fraction $\alpha$ of gas molecules will be diffusely reflected with a Maxwellian distribution at the wall temperature $T_{w}$, and the remaining fraction will undergo specular reflection. In the present study, $T_{w}=T_{0}$.

As the normal heat flux $\bar{q}_{y}$ is a constant in the whole domain, integration of Eq. (10) gives the temperature as

$$
\bar{T}=-\frac{2 \sqrt{2}}{5} \bar{q}_{y} \bar{y}-\frac{2}{5} \bar{\sigma}_{y y}-\frac{1}{5} \bar{R}_{y y}-\frac{1}{15} \bar{\Delta}+A,
$$

where $A$ is an integration constant. The superposition of the temperature contributions from $q_{y}, \sigma_{y y}, R_{y y}$, and $\Delta$ are clearly expressed by Eq. (27). This is an important feature that is not present in the NSF equations. The expressions for $\bar{q}_{y}, \bar{m}_{y y y}$, $\bar{R}_{y y}$, and $\bar{\Delta}$ can be obtained from Eqs. (9)-(14) as

$$
\begin{aligned}
\bar{\sigma}_{y y}= & G \bar{q}_{y}\left(0.6751 C_{1} e^{-1.4365 \bar{y}}+0.05799 C_{2} e^{-0.9601 \bar{y}}\right. \\
& \left.-0.1444 C_{3} e^{-2.4662 \bar{y}}\right), \\
m_{y y y}= & G \bar{q}_{y}\left(0.9969 C_{1} e^{-1.4365 \bar{y}}+0.1282 C_{2} e^{-0.9601 \bar{y}}\right. \\
& \left.-0.1242 C_{3} e^{-2.4662 \bar{y}}\right), \\
\bar{R}_{y y}= & G \bar{q}_{y}\left(C_{1} e^{-1.4365 \bar{y}}+C_{2} e^{-0.9601 \bar{y}}+C_{3} e^{-2.4662 \bar{y}}\right), \\
\Delta= & G \bar{q}_{y}\left(-4.5477 C_{1} e^{-1.4365 \bar{y}}+4.3399 C_{2} e^{-0.9601 \bar{y}}\right. \\
& \left.-2.1738 C_{3} e^{-2.4662 \bar{y}}\right),
\end{aligned}
$$


where $C_{1}, C_{2}$, and $C_{3}$ are integration constants. In the solutions given by Eqs. (28)-(31), the boundary condition that as $\bar{y} \rightarrow \infty, \bar{\sigma}_{y y}, \bar{m}_{y y y}, \bar{R}_{y y}$, and $\bar{\Delta}$ will remain finite has been used to remove the other three integration constants and the terms associated with them. The remaining integration constants $A, C_{1}, C_{2}$, and $C_{3}$ are determined from the wall boundary conditions (22) $-(26)$ by

$$
\begin{aligned}
A & =G \bar{q}_{y} C_{4} \\
C_{1} & =-\frac{1}{D}\left(0.0531 G^{2}+0.115 G+0.063\right) \\
C_{2} & =-\frac{1}{D}\left(0.3658 G^{2}+0.8979 G+0.5392\right) \\
C_{3} & =\frac{1}{D}\left(0.0839 G^{2}+0.1636 G+0.0644\right) \\
C_{4} & =-\frac{1}{D}\left(0.2757 G^{3}+1.1545 G^{2}+1.5748 G+0.6991\right)
\end{aligned}
$$

with $D=0.5513 G^{3}+2.1059 G^{2}+2.653 G+1.1003$. Inserting Eqs. (29), (30), and (32) into Eq. (27), the final expression for temperature from the LR26 moment equations reads

$$
\begin{aligned}
\bar{T}= & -\frac{2 \sqrt{2}}{5} \bar{q}_{y} \bar{y}-G \bar{q}_{y}\left(0.16686 C_{1} e^{-1.4365 \bar{y}}\right. \\
& \left.+0.5125 C_{2} e^{-0.9601 \bar{y}}-0.00268 C_{3} e^{-2.4662 \bar{y}}-C_{4}\right) .
\end{aligned}
$$

In the LR13 system, the governing differential equations (11)-(13) are replaced by the following constitutive relationships $[6,8]$ :

$$
\begin{aligned}
\bar{m}_{y y y} & =-\frac{6}{5} \frac{\lambda}{L} \sqrt{\frac{2}{\pi}} \frac{\partial \bar{\sigma}_{y y}}{\partial \bar{y}}, \\
\bar{R}_{y y} & =-\frac{16}{5} \frac{\lambda}{L} \sqrt{\frac{2}{\pi}} \frac{\partial \bar{q}_{y}}{\partial \bar{y}}=0,
\end{aligned}
$$

and

$$
\bar{\Delta}=-12 \frac{\lambda}{L} \sqrt{\frac{2}{\pi}} \frac{\partial \bar{q}_{y}}{\partial \bar{y}}=0,
$$

along with the boundary conditions (22) and (23) without the higher moment $\bar{\phi}_{\text {yyyy }}$. For the temperature-jump problem, the temperature field for the LR13 equations is readily obtained as

$$
\begin{aligned}
\bar{T}= & -\frac{2 \sqrt{2}}{5} \bar{q}_{y} \bar{y} \\
& +G \bar{q}_{y}\left[\frac{4 \sqrt{30 \pi}}{75(\sqrt{60}+4 G)} e^{-\sqrt{15} \bar{y} / 2}-\frac{(13 \sqrt{30}+50 G)}{25(\sqrt{30}+4 G)}\right] .
\end{aligned}
$$

If we compare equations (37) and (41), we can see that the LR26 equations provide three exponentials to describe the thermal Knudsen layer, while the LR13 equations only contain one exponential term.

\section{TEMPERATURE DEFECT AND THE TEMPERATURE-JUMP COEFFICIENT}

In kinetic theory, the temperature defect and temperaturejump coefficient are often used to study how the wall affects the temperature profile. The normalized temperature disturbance, $\hat{T}$, from the equilibrium temperature, $T_{0}$, defined by

$$
\hat{T}=-\frac{\kappa\left(T-T_{0}\right)}{L q_{y}}
$$

were obtained and studied in kinetic theory with the linearized Boltzmann equation [13-16]. With Eqs. (17)-(19), $\hat{T}$ can be expressed by

$$
\hat{T}=-\frac{5 \bar{T}}{2 \sqrt{2} \bar{q}_{y}} .
$$

The temperature $\hat{T}$ from the LR26 equations can be written as

$$
\begin{aligned}
\hat{T}= & \bar{y}+\frac{5 \sqrt{2} G}{4}\left(0.1669 C_{1} e^{-1.4365 \bar{y}}+0.5125 C_{2} e^{-0.9601 \bar{y}}\right. \\
& \left.-0.00268 C_{3} e^{-2.4662 \bar{y}}-C_{4}\right) .
\end{aligned}
$$

A temperature defect, $T_{d}$, is defined by [13-16]

$$
T_{d}=\bar{y}+\eta-\hat{T},
$$

and the temperature-jump coefficient $\eta$ is determined through

$$
\lim _{\bar{y} \rightarrow \infty} T_{d}=0 .
$$

From the above condition, the expression for $\eta$ from the LR26 equations can be written as

$$
\eta=-\frac{5 \sqrt{2} G}{4} C_{4}
$$

and the temperature defect is expressed by

$$
\begin{aligned}
T_{d}= & -\frac{5 \sqrt{2} G}{4}\left(0.1669 C_{1} e^{-1.4365 \bar{y}}+0.5125 C_{2} e^{-0.9601 \bar{y}}\right. \\
& \left.-0.00268 C_{3} e^{-2.4662 \bar{y}}\right) .
\end{aligned}
$$

Similarly, we can obtain the temperature defect and associated jump coefficient for the LR13 equations, respectively, as

$$
T_{d}=G \frac{2 \sqrt{15}}{15(\sqrt{30}+4 G)} e^{-\sqrt{15} \bar{y} / 2}
$$

and

$$
\eta=G \frac{(13 \sqrt{15}+25 G \sqrt{2})}{10(\sqrt{30}+4 G)} .
$$

\section{RESULTS AND COMPARISON WITH KINETIC THEORY}

The temperature-jump problem is among one of the most fundamental problems of rarefied gas dynamics. It has been used as one of the test cases for numerous analytical and numerical approaches. Loyalka [14] investigated the temperature-jump problem with a hard sphere gas based on the linearized Boltzmann equation. Barichello and Siewert [15] 
used the discrete-ordinates method to solve the temperaturejump problem based on the Bhatnagar-Gross-Krook (BGK) model. In the work by Barichello et al. [16], the Boltzmann equation was solved using three different kinetic models to determine the Knudsen layer temperature profile close to the wall. These included the BGK model, the Williams model (the collision frequency is proportional to the magnitude of the velocity), and the hard sphere model. They investigated wall conditions ranging from fully diffusive to specular-diffusive reflection. However, the authors are not aware of any DSMC studies for this classic problem which may be attributed to the complexity of setting up the free boundary condition for this half-space problem. In our results, we consider only Maxwell molecules for ease of the mathematical treatment. Figure 1 presents the analytical solutions of the temperature defect in the Knudsen layer obtained from the moment equations in comparison with the computational results from the three kinetic models for the case of $\alpha=1,0.5,0.1$. As shown in Fig. 1(a) for fully diffusive reflection at the wall, the BGK kinetic model produces a larger temperature defect in the Knudsen layer than the hard sphere model. The analytical solution from the LR26 equations generally lies between the two models and is in close agreement with the BGK model. Conversely, the solution obtained from the LR13 equations consistently underpredicts the temperature defect, as shown in Fig. 1(a). As the LR13 system involves fewer equations and boundary conditions than the LR26 system, less kinetic information is preserved in the LR13 model. In the NSF equations, kinetic information is lost, so there is no thermal Knudsen layer captured. As the value of $\alpha$ reduces to 0.5 , which corresponds to a half-specular and half-diffusive reflection condition, the temperature defect becomes more profound and the Knudsen layer gets wider, as indicated in Fig. 1(b). The BGK kinetic model produces the largest temperature defect in the Knudsen layer, particularly close to the wall $(\bar{y}<0.5)$. In contrast, the results from the Williams and hard sphere models are in close agreement with each other. The LR26 equations follow the kinetic data as the wall boundary changes from diffusive to specular reflection. The analytical solution from the LR26 equations generally lies between the three models. Even when the value of $\alpha$ reduces further to 0.1 , the LR26 equations still follow the kinetic data. In contrast, the solution obtained from the LR13 equations begins to significantly underpredict the temperature defect, particularly at very low values of $\alpha$, as shown in Figs. 1(b) and 1(c). Clearly the combination of three exponentials with different widths produces an improved thermal Knudsen layer temperature profile. It is expected that more moments and their governing equations would generate more exponentials with different widths to fully recover the full kinetic information. Equation (27) illustrates that the temperature in the thermal Knudsen layer consists of contributions from $\bar{\sigma}_{y y}, \bar{R}_{y y}$, and $\bar{\Delta}$. For the LR13 equations, there is no mechanism for $\bar{R}_{y y}$ and $\bar{\Delta}$ to contribute; so the Knudsen layer is derived solely from the normal stress, $\bar{\sigma}_{y y}$. In the NSF system, $\bar{\sigma}_{y y}$ is not present, so there is no thermal Knudsen layer.

A further test of the ability of macroscopic models to accurately capture the nonequilibrium features in the thermal Knudsen layer is an evaluation of the temperature-jump coefficient. It is appropriate to plot $\eta / \alpha^{*}$ against $\alpha$ to gain
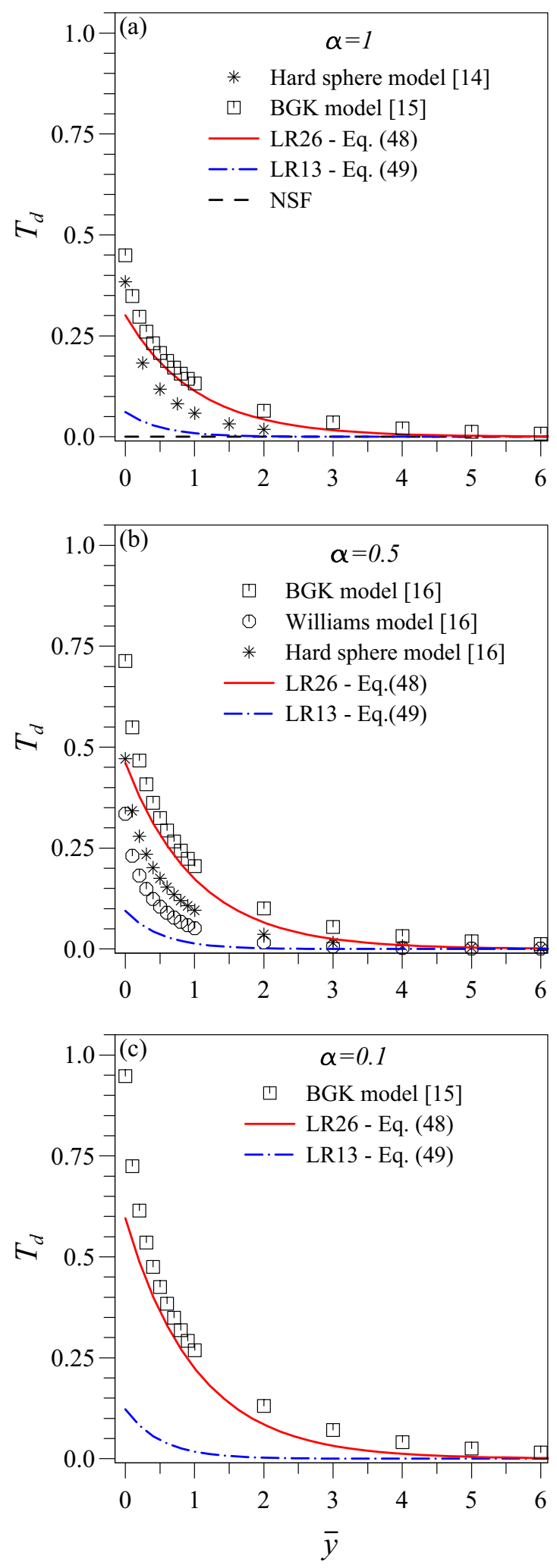

FIG. 1. (Color online) Temperature defect profiles: Comparison between the linearized-moment solutions (lines) and kinetic theory (symbols) [14-16] for different values of accommodation coefficient.

a better understanding of the accuracy of each equation set against kinetic data, as shown in Fig. 2. The temperature-jump coefficient predicted by the BGK model is greater than the 


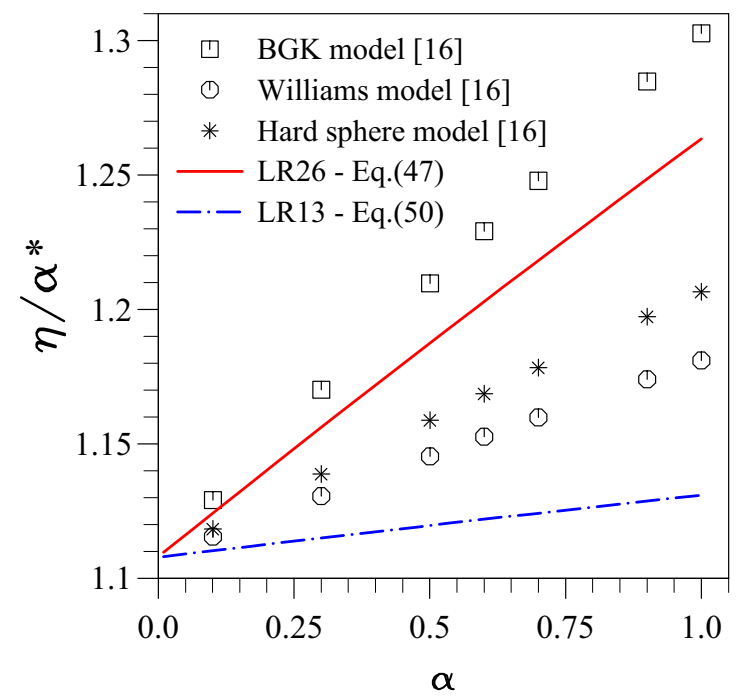

FIG. 2. (Color online) Temperature-jump coefficient predicted by the linearized-moment equations (lines) in comparison with kinetic theory (symbols) [16]. [Note: $\alpha^{*}=(2-\alpha) / \alpha$.]

value from either the Williams or hard sphere models and the solution from the LR26 equations lies between them, providing excellent qualitative agreement. In contrast, the LR13 solution underestimates the value of the temperaturejump coefficient due to the information lost in the reduction of the moment equations. The temperature-jump coefficient is often used as a boundary condition for the NSF equations to compensate for their inability to describe thermal Knudsen layers. However, the value of the temperature-jump coefficient determined from this classic thermal problem clearly depends on the kinetic model used and might only be applicable to planar geometry. In situations involving more complex geometries and flow conditions, care must be taken when using any temperature-jump coefficient in NSF calculations.
In the hydrodynamic limit, the BGK model has a Prandtl number of unity, whereas Maxwell molecules and the hard sphere gas model have a value of $\operatorname{Pr}=2 / 3$. However, in the Knudsen layer, the traditional gradient transport mechanism is no longer valid. To improve the predictive capability of macroscopic models the concept of an effective viscosity, $\mu_{\mathrm{eff}}$, near a solid wall has often been explored. This allows design engineers to extend the NSF equations into the nonequilibrium flow regime [19-22] to evaluate mean quantities of interest. For example, in Kramers' problem, the effective viscosity $\mu_{\text {eff }}$ is defined as [18]

$$
\sigma_{x y}=-\mu_{\mathrm{eff}} \frac{\partial u}{\partial y}
$$

where $\sigma_{x y}$ is the shear stress. From the LR26 equations for Maxwell molecules, the ratio of $\mu_{\mathrm{eff}}$ to $\mu$ is given by [18]

$$
\begin{aligned}
\frac{\mu_{\mathrm{eff}}}{\mu}= & {\left[1-\left(1.3042 B_{1} e^{-(1.265 \sqrt{\pi / 2}) \hat{y}}\right.\right.} \\
& \left.\left.+1.6751 B_{2} e^{-(0.5102 \sqrt{\pi / 2}) \hat{y}}\right)\right]^{-1} .
\end{aligned}
$$

Here, $\hat{y}$ is the distance perpendicular to the wall normalized by the mean free path $\lambda$, and $\hat{y}=(L / \lambda) \bar{y}$. The coefficients $B_{1}$ and $B_{2}$ are given by [18]

$$
\begin{aligned}
& B_{1}=-\frac{2-\alpha}{\alpha} \frac{8.1265 \alpha^{2}+128.24 \alpha}{0.48517 \alpha^{2}+64.884 \alpha+809.95}, \\
& B_{2}=-\frac{2-\alpha}{\alpha} \frac{0.08565 \alpha^{2}+36.2 \alpha}{0.48517 \alpha^{2}+64.884 \alpha+809.95} .
\end{aligned}
$$

In a similar manner, the effective thermal conductivity $\kappa_{\text {eff }}$ in the Knudsen layer can be defined as

$$
q_{y}=-\kappa_{\mathrm{eff}} \frac{\partial T}{\partial y} \text {. }
$$

From Eqs. (55) and (37), the ratio of $\kappa_{\text {eff }}$ to $\kappa$ is readily obtained for Maxwell molecules by

$$
\begin{aligned}
\frac{\kappa_{\mathrm{eff}}}{\kappa} & =-\frac{2 \sqrt{2}}{5} \frac{\bar{q}_{y}}{\partial \bar{T} / \partial \bar{y}}=\left[1-\frac{2 \sqrt{2}}{5} G\left(0.2397 C_{1} e^{-1.4365 \bar{y}}+0.4921 C_{2} e^{-0.9601 \bar{y}}-0.006556 C_{3} e^{-2.4662 \bar{y}}\right)\right]^{-1} \\
& =\left[1-\frac{2 \sqrt{2}}{5} G\left(0.2397 C_{1} e^{-0.4778 \sqrt{\pi} \hat{y}}+0.4921 C_{2} e^{-0.32 \sqrt{\pi} \hat{y}}-0.006556 C_{3} e^{-0.8221 \sqrt{\pi} \hat{y}}\right)\right]^{-1} .
\end{aligned}
$$

The effect of the wall and the accommodation coefficient on the effective thermal conductivity is shown in Fig. 3, which shows that $\kappa_{\text {eff }}$ reduces as the gas approaches the wall. A smooth wall with a small accommodation coefficient will reduce the value of $\kappa_{\text {eff }}$ further and a similar near-wall effect has been observed for the effective viscosity $\mu_{\text {eff }}[18,20-22]$. However, it should be noted that the effective viscosity and thermal conductivity can only be used as a phenomenological model to mimic the Knudsen layer velocity and temperature profile for the NSF equations in a similar configuration. When flow conditions or the geometrical configuration change, the effective viscosity and thermal conductivity are likely to change as well, since they are not properties of the fluid and dependent on the flow conditions.

The impact of the wall on the ratio of momentum diffusivity to thermal diffusivity can be examined by the effective Prandtl number, $\operatorname{Pr}_{\text {eff }}$, in the Knudsen layer. Analogous to Eq. (20), the effective Prandtl number can be defined as

$$
\operatorname{Pr}_{\mathrm{eff}}=\frac{5}{2} \frac{R \mu_{\mathrm{eff}}}{\kappa_{\mathrm{eff}}}=\operatorname{Pr}\left[\frac{1-2 \sqrt{2} / 5 G\left(0.2397 C_{1} e^{-0.4778 \sqrt{\pi} \hat{y}}+0.4921 C_{2} e^{-0.32 \sqrt{\pi} \hat{y}}-0.006556 C_{3} e^{-0.8221 \sqrt{\pi} \hat{y}}\right)}{1-\left(1.3042 B_{1} e^{-(1.265 \sqrt{\pi / 2}) \hat{y}}+1.6751 B_{2} e^{-(0.5102 \sqrt{\pi / 2}) \hat{y}}\right)}\right] .
$$




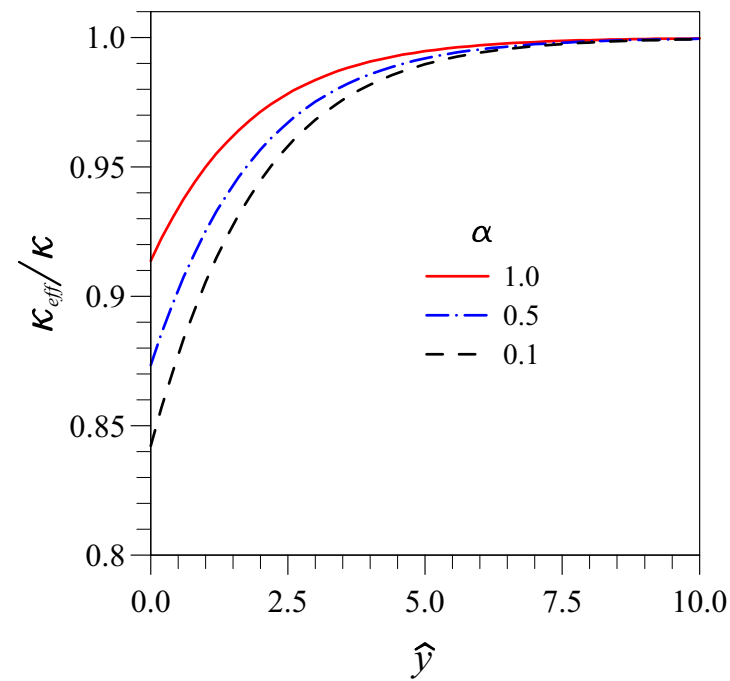

FIG. 3. (Color online) The wall effect on the effective thermal conductivity in the Knudsen layer.

Profiles of the effective Prandtl number in the near-wall region for different accommodation coefficients are presented in Fig. 4. The effective Prandtl number is significantly reduced from its hydrodynamic value as the gas approaches the wall. Below a distance of $1.85 \lambda$, nonequilibrium effects introduced by the wall enhances thermal conduction rather than convection. When the gas is more than $1.85 \lambda$ from the wall, the effective Prandtl number is slightly above its hydrodynamic value and gradually approaches its hydrodynamic limit further away from the wall. The smoother the wall, the more significant the phenomenon exhibited.

\section{CONCLUSIONS}

The moment method has been employed to study a classic thermal problem. Analytical solutions for the temperature defect and temperature-jump coefficient have been obtained for the linearized 13 and 26 moment equations, respectively. In comparison with data from kinetic theory, it is found

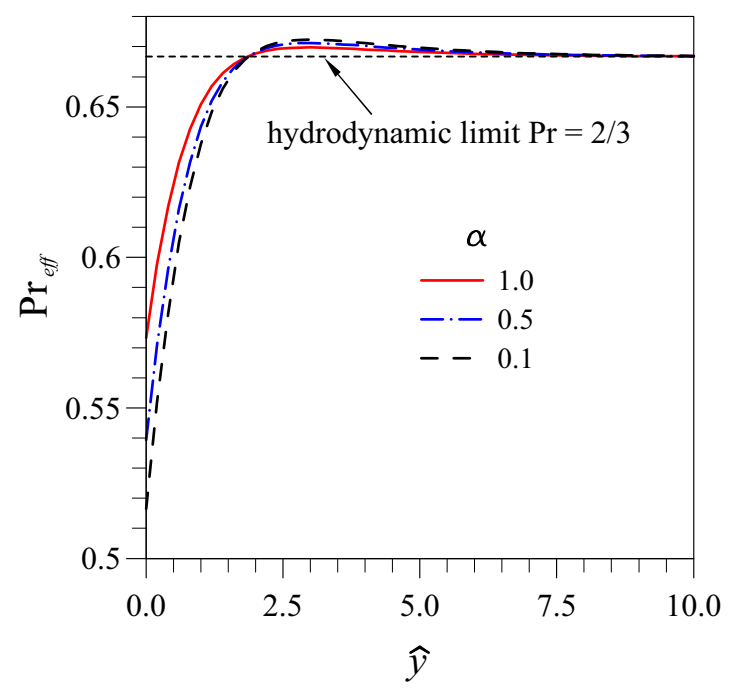

FIG. 4. (Color online) The effective Prandtl number in the Knudsen layer.

that the linearized 26 moment equations provide an excellent qualitative description of the thermal Knudsen layer in terms of the temperature defect and the temperature-jump coefficient, while the linearized 13 moment equations consistently underpredict the kinetic data. Moreover, it was shown that the linearized 26 moment equations can be applied to wall boundaries dominated by either specular or diffusive reflection and the results indicate that the physics of nonequilibrium gas flow can be successfully captured by using higher-order moment equations. Expressions for the effective thermal conductivity and the effective Prandtl number are derived for Maxwell molecules and evaluated in the half-space configuration with the linearized 26 moment equations which help to illustrate the complex behavior of the near-wall thermal Knudsen layer.

\section{ACKNOWLEDGMENT}

The authors would like to thank the Engineering and Physical Science Research Council (EPSRC) for their support of Collaborative Computational Project 12 (CCP12).
[1] C. Cercignani, The Boltzmann Equation and Its Applications (Springer, New York, 1988).

[2] G. Bird, Molecular Gas Dynamics and the Direct Simulation of Gas Flows (Clarendon, Oxford, 1994).

[3] H. Grad, Commun. Pure Appl. Math. 2, 331 (1949).

[4] H. Struchtrup, Macroscopic Transport Equations for Rarefied Gas Flows (Springer, Berlin, 2005).

[5] X. J. Gu and D. R. Emerson, J. Comput. Phys. 225, 263 (2007).

[6] M. Torrilhon and H. Struchtrup, J. Comput. Phys. 227, 1982 (2008).

[7] X. J. Gu and D. R. Emerson, J. Fluid Mech. 636, 177 (2009).

[8] P. Taheri, M. Torrilhon, and H. Struchtrup, Phys. Fluids 21, 017102 (2009).
[9] X. J. Gu, D. R. Emerson, and G. H. Tang, Phys. Rev. E 81, 016313 (2010).

[10] X. J. Gu and D. R. Emerson, Microfluid. Nanofluid. 10, 389 (2011).

[11] J. B. Young, Int. J. Heat Mass Transfer 54, 2902 (2011).

[12] M. Smoluchowski, Ann. Phys. (Leipzig) 35, 983 (1911).

[13] S. K. Loyalka, C. E. Siewert, and J. R. Thomas, Jr., Phys. Fluids 21, 854 (1978).

[14] S. K. Loyalka, Phys. Fluids A 1, 403 (1989).

[15] L. B. Barichello and C. E. Siewert, Eur. J. Appl. Math. 11, 353 (2000).

[16] L. B. Barichello, A. C. R. Bartz, M. Camargo, and C. E. Siewert, Phys. Fluids 14, 382 (2002). 
[17] H. Struchtrup and M. Torrilhon, Phys. Fluids 15, 2668 (2003).

[18] X. J. Gu, D. R. Emerson, and G. H. Tang, Continuum Mech. Thermodyn. 21, 345 (2009).

[19] T. Veijola, H. Kuisma, J. Lahdenperä, and T. Ryhänen, Sens. Actuators, A 48, 239 (1995).
[20] Z. L. Guo, B. C. Shi, and C. G. Zheng, Europhys. Lett. 80, 24001 (2007).

[21] D. A. Lockerby and J. M. Reese, J. Fluid Mech. 604, 235 (2008).

[22] G. H. Tang, Y. H. Zhang, X. J. Gu, and D. R. Emerson, Europhys. Lett. 83, 40008 (2008). 